\title{
Bem-estar espiritual e transtornos psiquiátricos menores em estudantes de Psicologia: estudo transversal
}

\author{
Marina Bento Gastaud* \\ Luciano Dias de Mattos Souza** \\ Laryssa Braga* \\ Cristina Lessa Horta*** \\ Flávio Martinez de Oliveira*** \\ Paulo Luis Rosa Sousa \\ Ricardo Azevedo da Silva****
}

\section{INTRODUÇÃO}

A religiosidade e a espiritualidade sempre foram consideradas importantes aliadas das pessoas que sofrem e/ou estão doentes. Entretanto, a medicina ocidental como um todo e a psiquiatria, em especial, têm tido duas posturas básicas em relação ao tema: 1) negligência, por considerar esses assuntos irrelevantes ou fora de sua área de interesse principal; 2) oposição, ao caracterizar as experiências religiosas de seus pacientes como evidências de psicopatologias diversas ${ }^{1,2}$.

De acordo com a Organização Mundial da Saúde, $24 \%$ da clientela dos centros de atenção primária apresenta transtornos psiquiátricos, como depressão, ansiedade e uso inadequado de substâncias $^{3}$. Na população urbana de

\footnotetext{
*Acadêmicos, Escola de Psicologia, Universidade Católica de Pelotas, Pelotas, RS

** Mestrando em Saúde e Comportamento, Universidade Católica de Pelotas, Pelotas, RS.

*** Professores, Universidade Católica de Pelotas, Pelotas, RS.

**** Professor titular, Faculdade de Psicologia, e orientador, Mestrado em Saúde e Comportamento, Universidade Católica de Pelotas, Pelotas, RS.
}

Pelotas, foi encontrada uma prevalência de $22,7 \%$ de transtornos psiquiátricos menores (TPM) em maiores de 15 anos $^{4}$. Há, portanto, a necessidade de desenvolver novas formas de promover a saúde mental na população, formas que devem considerar também a dimensão "espiritual", tendência que se mostra em expansão ${ }^{5}$.

A questão da espiritualidade é muito ampla, e sua mensuração, bastante complexa, sendo o bem-estar espiritual (SWB) um de seus aspectos passíveis de avaliação. Por SWB, entende-se a percepção subjetiva de bem-estar do sujeito em relação à sua crença. $O$ desenvolvimento de instrumentos de mensuração do SWB foi baseado no conceito de espiritualidade que envolve um componente vertical, religioso (um sentido de bem-estar em relação a Deus), e um componente horizontal, existencial (um sentido de propósito e satisfação de vida), sendo que este último não implica em qualquer referência a conteúdo especificamente religioso ${ }^{6}$. A espiritualidade coloca questões a respeito do significado da vida e da razão de viver, não se limitando a alguns tipos de crenças ou práticas. A religião é 
definida como a crença na existência de um poder sobrenatural, criador e controlador do universo, que deu ao homem uma natureza espiritual que continua a existir depois da morte do seu corpo. Religiosidade é a extensão na qual um indivíduo acredita, segue e pratica uma religião.

A influência da espiritualidade na saúde física, mental e social tem sido amplamente demonstrada ${ }^{3,7,8}$. Há evidências crescentes de que a religiosidade (uma das dimensões da espiritualidade) está associada com saúde mental. Sabe-se que a presença de religiosidade pode ser tanto um fator protetor como desagregador para a saúde mental. Em um estudo de revisão, foi mostrada uma associação positiva em $50 \%$ dos casos e negativa em $25 \%$ deles. Nessa revisão, a religiosidade foi considerada como sendo um fator protetor para o suicídio, abuso de drogas e álcool, comportamento delinqüente, satisfação marital, sofrimento psicológico e alguns diagnósticos de psicoses funcionais ${ }^{9}$. Volcan et al. também mostraram que o SWB atua como fator associado para TPM, já que indivíduos com SWB baixo e moderado manifestaram o dobro de chances de apresentar tais transtornos.

Apesar da associação entre saúde mental e religiosidade, existe uma disparidade entre as crenças dos profissionais da área psicológica e psiquiátrica e as crenças da população em geral. Enquanto cerca de $90 \%$ da população em geral acredita em Deus, apenas $43 \%$ de uma amostra de psiquiatras membros da Associação Psiquiátrica Americana tem tal crença $^{10}$.

Há uma escassez na literatura de estudos que envolvam religiosidade com estudantes de Psicologia. Um estudo recente encontrado foi o de Lewis, com 48 estudantes de Psicologia, na Irlanda. Nesse estudo, encontrou-se que a religiosidade é percebida como sendo acompanhada por níveis altos de obsessividade, sugerindo a existência de um estereótipo ou de um pré-conceito em relação aos efeitos da religião na saúde mental entre alunos do curso de Psicologia ${ }^{11}$.

Os achados sugerem que religiosidade e saúde mental estão positivamente associadas $^{11}$, e que o fortalecimento do SWB pode auxiliar significativamente na redução da angústia relacionada a doenças e na promoção da saúde ${ }^{6}$. Entretanto, há a hipótese de que profissionais e estudantes promotores da saúde mental teriam um escore reduzido na escala de SWB (SWBS).
O presente trabalho foi desenvolvido em continuidade a nosso estudo anterior ${ }^{6}$ para: 1 ) testar a hipótese de que a atividade psicológica associa-se a reduzido SWB; e 2) verificar se a relação entre saúde mental e religiosidade sugerida na literatura é extensiva também aos alunos do curso de Psicologia.

\section{MÉTODOS}

Durante o ano de 2002, foi entrevistada a totalidade dos alunos do curso de Psicologia da Universidade Católica de Pelotas (RS) ( $\mathrm{n}=$ 351). A coleta de dados foi realizada por uma única acadêmica do curso de Psicologia. Foi utilizado um questionário auto-aplicável contendo a SWBS, de Paloutzian \& Ellison, SelfReporting Questionnaire (SRQ-20) e informações sociodemográficas.

A SWBS é constituída de 20 itens, dos quais 10 avaliam o bem-estar religioso (RWB), e os demais, o bem-estar existencial (EWB). Cada um dos 20 itens é respondido em uma escala de 6 pontos, a qual varia de "concordo fortemente" a "discordo fortemente". O examinando deve graduar afirmações do tipo "Eu acredito que Deus está preocupado com meus problemas" para RWB e do tipo "Eu me sinto realizado e satisfeito com a vida" para EWB. Os escores das duas subescalas são somados para obtenção da medida geral de SWB. Testes de confiabilidade, obtidos em uma amostra de 100 estudantes voluntários da Universidade de Idaho, tiveram como resultados os coeficientes de 0,93 (SWB), 0,96 (RWB) e 0,86 (EWB). Como índice de consistência interna, os coeficientes alfa encontrados foram 0,89 para o índice geral, 0,87 para subescala de RWB e 0,78 para subescala de EWB. A magnitude desses coeficientes sugere que a SWBS tem alta confiabilidade e consistência interna. Os escores da SWBS estão correlacionados, como esperado, a outras medidas de avaliação da espiritualidade/religiosidade, bem como a outros índices de bem-estar. Paloutzian \& Ellison sugerem como pontos de corte para 0 escore geral de SWB os intervalos de 20 a 40, 41 a 99 e 100 a 120, para baixo, moderado e alto SWB, respectivamente. Nas duas subescalas, os intervalos são de 10 a 20, 21 a 49 e 50 a 60 pontos $^{12}$. Na análise desse estudo, os resultados da SWBS foram denominados positivo para escore alto e negativo para escores moderado e baixo.

Optou-se pela utilização desse instrumento porque ele possibilita a mensuração 
quantitativa da espiritualidade, diferenciando-a da dimensão estritamente religiosa, tendo sido adaptado para o Brasil por Volcan et al. ${ }^{6}$.

O SRQ-20 é um instrumento para levantamento de TPM recomendado pela OMS, validado para a população brasileira por Mari \& Williams $^{13}$. No presente estudo, considerou-se que os sujeitos do sexo masculino que obtivessem escores de até 7 pontos e os do sexo feminino com pontuações de até 6 pontos seriam considerados SRQ-20 negativo, enquanto que os que obtivessem pontuações acima destas teriam a denominação de SRQ-20 positivo.

A entrada de dados foi realizada utilizando o Epi Info 5.0, e a análise dos dados foi feita através do SPSS.

Inicialmente, os dados do curso de Psicologia foram organizados em tabelas que utilizaram freqüências relativas e freqüências percentuais. O teste do qui-quadrado foi usado para avaliar a significância da diferença entre esses resultados e os resultados relativos aos cursos de Direito e Medicina. Para avaliar a associação entre TPM e SWB, utilizou-se a regressão logística. A razão de chances foi calculada levando-se em conta duas possibilidades de resposta quanto ao SWB (negativo e positivo) em relação ao resultado do SRQ-20.
O projeto foi aprovado pelo Comitê de Ética em Pesquisa da Santa Casa de Pelotas.

\section{RESULTADOS}

Os dados obtidos com os alunos do curso de Psicologia foram comparados aos resultados encontrados por Volcan et al. ${ }^{6}$, em pesquisa anterior, que utilizou a mesma metodologia para comparar e descrever uma amostra de alunos dos cursos de Direito e Medicina da mesma universidade. A soma das amostragens incluiu o total de 815 alunos na análise.

$O$ estudo anteriormente realizado pesquisou 464 universitários, matriculados no terceiro, quarto e quinto anos dos cursos de Direito e Medicina, durante os meses de maio e junho de 2001. A coleta de dados foi realizada por psicólogos ou acadêmicos de psicologia, e os alunos ausentes foram procurados para responder individualmente ao questionário; destes, 43 não foram encontrados $(9,3 \%$ da amostra) ${ }^{6}$.

Quando foi agrupado o presente estudo com o estudo anterior, obteve-se uma amostra ( $n=815)$ que apresentou um predomínio do gênero masculino no curso de Medicina (58,8\%) e feminino nos cursos de Direito (55\%) e Psicologia (89\%) (tabela 1).

Tabela 1 - Distribuição da amostra quanto ao gênero e faixa etária

\begin{tabular}{lccccccc}
\hline & \multicolumn{2}{c}{ Medicina } & \multicolumn{2}{c}{ Direito } & \multicolumn{2}{c}{ Psicologia } & \\
\cline { 2 - 7 } & $n$ & $\%$ & $n$ & $\%$ & $n$ & $\%$ & \\
\hline Masculino & 97 & $58,8 \%$ & 109 & $45 \%$ & 37 & $11 \%$ & $\alpha=0,05$ \\
Feminino & 68 & $41,2 \%$ & 133 & $55 \%$ & 299 & $89 \%$ & $p<0,001$ \\
$19-25$ anos & 149 & $88,6 \%$ & 191 & $76,9 \%$ & 235 & $69,3 \%$ & $\alpha=0,05$ \\
26 anos ou mais & 19 & $11,4 \%$ & 58 & $23,1 \%$ & 104 & $30,7 \%$ & $p<0,001$ \\
\hline
\end{tabular}

A faixa etária dos grupos teve média de 24,4 anos; a idade dos alunos de Medicina variou de 20 a 33 anos; dos de Direito, de 19 a 59 anos; e dos de Psicologia, de 17 a 64 anos. Enquanto $30,7 \%$ dos alunos de Psicologia tinham idade superior ou igual a 26 anos, apenas $11,4 \%$ da amostra de Medicina encontrava-se nessa faixa etária $(p<0,001)$.

Quanto ao desempenho na SWBS, observou-se que $68,5 \%$ dos alunos de Direito e
$68,8 \%$ dos alunos de Medicina apresentaram escores negativos de espiritualidade, enquanto $84,6 \%$ dos alunos de Psicologia obtiveram resultado negativo nessa escala $(p<0,001)$. $\mathrm{Na}$ subescala de EWB, 35,9\% dos alunos de Medicina, 31,5\% dos alunos de Direito e 16,5\% dos acadêmicos de Psicologia demonstraram escores de EWB positivo (tabela 2).

Na variável RWB, houve maior semelhança entre os grupos. Obtiveram resultado positivo 
Bem-estar e transtornos menores em estudantes de Psicologia - Gastaud et al.

Tabela 2 - Distribuição da amostra quanto à SWBS e subescalas

\begin{tabular}{cccccccc}
\hline & \multicolumn{2}{c}{ Medicina } & \multicolumn{2}{c}{ Direito } & \multicolumn{2}{c}{ Psicologia } & \\
\cline { 2 - 6 } & $n$ & $\%$ & $n$ & $\%$ & $n$ & $\%$ & \\
\hline $\begin{array}{ccccccc}\text { Bem-estar espiritual } \\
+\end{array}$ & 53 & $31,2 \%$ & 79 & $31,5 \%$ & 53 & $15,4 \%$ & $\alpha=0,05$ \\
- & 117 & $68,8 \%$ & 172 & $68,5 \%$ & 292 & $84,6 \%$ & $p<0,001$ \\
Bem-estar existencial & & & & & & & \\
+ & 61 & $35,9 \%$ & 79 & $31,5 \%$ & 57 & $16,5 \%$ & $\alpha=0,05$ \\
+ & 109 & $64,1 \%$ & 172 & $68,5 \%$ & 288 & $83,5 \%$ & $p<0,001$ \\
Bem-estar religioso & & & & & & & \\
+ & 61 & $35,9 \%$ & 93 & $37,1 \%$ & 92 & $26,7 \%$ & $\alpha=0,05$ \\
+ & 109 & $64,1 \%$ & 158 & $62,9 \%$ & 253 & $73,3 \%$ & $0,01<p<0,05$ \\
\hline
\end{tabular}

Considerou-se positivo para escore alto e negativo para escores moderado e baixo.

35,9\% dos estudantes de Medicina, 37,1\% dos de Direito e $26,7 \%$ dos de Psicologia $(p<0,05)$.

No SRQ-20, não foram encontradas diferenças estatisticamente significativas entre os grupos, já que $21,1 \%$ dos acadêmicos de Psicologia obtiveram escores positivos, comparados a $17,5 \%$ dos alunos de Direito e $20 \%$ dos alunos de Medicina (tabela 3 ).

Tabela 3 - Distribuição da amostra quanto ao desempenho no SRQ-20

\begin{tabular}{cccccccc}
\hline & \multicolumn{2}{c}{ Medicina } & \multicolumn{2}{c}{ Direito } & \multicolumn{2}{c}{ Psicologia } & \\
\cline { 2 - 6 } & $n$ & $\%$ & $n$ & $\%$ & $n$ & $\%$ & \\
\hline SRQ-20 & & & & & & & \\
+ & 34 & $20 \%$ & 44 & $17,5 \%$ & 71 & $21,1 \%$ & $\alpha=0,05$ \\
- & 136 & $80 \%$ & 207 & $82,5 \%$ & 265 & $78,9 \%$ & $p>0,50$ \\
\hline
\end{tabular}

Considerou-se negativo para escores de até 7 pontos no sexo feminino e de até 6 pontos no sexo masculino. Entre os alunos de Psicologia, houve uma perda de $n=15$ nessa avaliação.

Nos alunos de Psicologia, não houve diferença estatisticamente significativa nas variáveis SRQ-20 e SWB quanto ao gênero. Conforme observado na tabela 4 , a razão de chances entre os níveis de SWB e o escore de SRQ-20 foi de 3,71 (IC 95\% 1,29-10,68), indicando que estudantes de Psicologia com TPM apresentam mais chances de obterem baixas pontuações na escala de bem-estar-espiritual e nas subescalas (existencial e religioso).

\section{DISCUSSÃO}

Os resultados do presente estudo indicam que estudantes de Psicologia com TPM apresentam mais chances de obterem baixas pontuações na escala de EWB. Indicam ainda que alunos de Psicologia com esses transtornos tiveram duas vezes mais chances de obterem baixas pontuações no RWB. Porém, nesse caso, não se pode afirmar que a relação é significativa (pois seu menor valor é 0,99 ), embora haja uma forte tendência (pois o intervalo engloba o 1).

Esses achados confirmam a hipótese de que estudantes do curso de Psicologia obteriam escores menores de SWB, existencial e religioso do que acadêmicos dos cursos de Direito e Medicina.

Quanto às diferenças na dimensão religiosa da escala, a experiência internacional mostra 
Bem-estar e transtornos menores em estudantes de Psicologia - Gastaud et al.

Tabela 4 - Associação entre bem-estar espiritual e suas subescalas com saúde mental nos estudantes de Psicologia

\begin{tabular}{cccc}
\hline & $n$ & Razão de chances SRQ-20 + & IC 95\% \\
\hline Bem-estar espiritual & & & \\
Negativo & 51 & 3,71 & \\
Positivo & 279 & Referência & $1,29-10,68$ \\
$\begin{array}{c}\text { Bem-estar religioso } \\
\text { Negativo }\end{array}$ & 90 & 2,05 & \\
Positivo & 240 & Referência & $0,99-4,25$ \\
Bem-estar existencial & & & \\
Negativo & 55 & 4,43 & \\
Positivo & 275 & Referência & $1,33-14,69$ \\
\hline
\end{tabular}

Os resultados apontam para uma relação inversa entre níveis de bem-estar e escores no SRQ.

justamente que psicólogos americanos, por exemplo, acreditam menos em Deus do que a população em geral. O instituto Gallup encontrou que $96 \%$ dos americanos acreditam em Deus, enquanto $26 \%$ dos psicólogos clínicos são ateus ou agnósticos ${ }^{14}$.

Ademais, percebe-se que ainda é muito presente no corpo docente do curso de Psicologia o distanciamento forte entre religião e ciência, já que elas parecem "existir em esferas separadas ou com pouca intersecção"15. Especula-se que a visão de Freud de que a religião seria para a vida social o que a neurose é para a vida individual ${ }^{16}$ ainda pode ser considerada um dos fatores de peso para a existência dessa diferença nos escores marcados entre os cursos.

Em contraposição, estudos recentes sugerem que a religião cumpre duas tarefas culturais e psicológicas fundamentais: possibilita a construção de um mundo possível, de uma ordem plausível e aceitável, dando um sentido ao caos fenomênico da experiência; e permite ao homem dar um sentido a seu sofrimento. Assim, numa perspectiva das ciências sociais e psicológicas contemporâneas, a religião não é mais vista como simples sistema defensivo ou de alienação. Seu papel como instituição social, organizador da experiência subjetiva, tem sido enfatizado tanto por cientistas sociais quanto por pesquisadores das áreas de saúde mental ${ }^{17}$. Tal idéia confirmaria o achado de que, nessa amostra, a presença de TPM associa-se a baixos escores de RWB.
A amostra dos três cursos apresentou uma menor prevalência de TPM que estudos de base populacional anteriormente publicados ${ }^{4}$. Entre os possíveis motivos para essa diferença, estarão 1) a concentração de sujeitos com melhor recurso socioeconômico freqüentando uma universidade privada, 2) o possível conhecimento prévio da escala pelos alunos de Medicina e Psicologia e 3) o conhecimento de psicopatologia pelos alunos da área da saúde, na medida em que a maior consciência dos próprios problemas os levaria a buscar recursos de tratamento. No entanto, não houve diferenças significativas quanto à prevalência de TPM entre os cursos, indicando uma homogeneidade da amostra. Tal achado confirma o argumento de que o fator socioeconômico seria o de maior força nessa questão.

Há cada vez mais evidências de que o SWB associa-se com as condições de saúde. Vários estudos sugerem que o exercício de atividades espirituais (a oração e outros rituais, por exemplo) pode influenciar psicodinamicamente, através de emoções positivas como a esperança, o perdão, a autoestima e o amor. Estas emoções podem ser importantes para a saúde mental, através da ação de mecanismos psiconeuroimunológicos e psicofisiológicos ${ }^{9}$.

Embora religiosidade e espiritualidade difiram-se pela clara sugestão por parte da primeira de um sistema de adoração e doutrina específica que é partilhada por um grupo ${ }^{18}$, há uma considerável sobreposição dessas noções, 
na medida em que altos níveis de RWB tendem a levantar os escores de EWB, por darem um sentido para a vida e para a existência.

Portanto, mesmo que contribuam para o entendimento da questão, os resultados obtidos não possibilitam o estabelecimento de relações causais, devido a limitações próprias do delineamento transversal.

\section{CONCLUSÕES}

Os achados sugerem que o SWB se constitui num fator inversamente correlacionado à TPM. Estudantes com alta pontuação no SRQ20 apresentaram mais chances de pontuarem menores escores na SWBS.

Ademais, fica evidenciado, na amostra, que as questões a respeito do significado da vida e da razão de viver (averiguadas através do EWB) são percebidas como menos satisfatórias pelos estudantes de Psicologia do que pelos estudantes dos outros dois cursos. Este achado pode ser preocupante na medida em que se supõe que os estudantes de Psicologia seriam, justamente, aqueles que deveriam estar mais voltados a essas questões de natureza existencial, tanto para si próprios como para seus pacientes. Possivelmente, este é um ponto de investigação para os coordenadores dos cursos de Psicologia, com respeito à natureza das influências sociais, culturais e psicológicas que os alunos - futuros profissionais - sofrem durante o período universitário. Quais fatores específicos podem associar-se com o desenvolvimento ou inibição da espiritualidade em alunos de Psicologia?

\section{REFERÊNCIAS}

1. Pargament KI, Brant CR. Religion and coping. In: Koenig $\mathrm{HG}$, ed. Handbook of religion and mental health. London: Academic Press; 1998. p. 111-28.

2. Sims A. Psyche - Spirit as well as mind? Br J Psychiatry. $1994 ; 165(4): 441-6$.

3. World Health Organization. Division of mental health and prevention of substance abuse. WHOQOL and spirituality, religiousness and personal beliefs (SRPB) - report on WHO Consultation. Geneve: WHO; 1998.

4. Lima M, Soares BGO, Mari JJ. Saúde e doença mental em Pelotas, RS: dados de um estudo populacional. Rev Psiquiatr Clin. 1999;26(5):225-35.

5. American Psychiatric Association. Diretrizes para o tratamento de transtornos psiquiátricos. Porto Alegre: Artmed; 2004.

6. Volcan SM, Sousa PL, Mari JJ, Horta B. Relação entre bem-estar espiritual e transtornos psiquiátricos menores: estudo transversal. Rev Saude Publica. $2003 ; 37(4): 440-5$.
7. Lotufo Neto F. Psiquiatria e religião: a prevalência de transtornos mentais entre ministros religiosos [tese de livre-docência]. São Paulo: Universidade Federal de São Paulo; 1997.

8. Sousa PLR, Tillmann IA, Horta CL, Oliveira FM. A religiosidade e suas interfaces com a medicina, a psicologia e a educação: o estado de arte. Psiq Prat Med. 2001;34(4):112-7.

9. Levin, JS, Chatters LM. Research on religion and mental health: an overview of empirical findings and theoretical issues. In: Koenig HG, ed. Handbook of religion and mental health. London: Academic Press; 1998. p.33-50.

10. Larson DB, Pattison EM, Blazer DG, Omram AR, Kaplan $\mathrm{BH}$. Systematic analysis of research on religion variables in four major psychiatric journals, 1978-1982. Am J Psychiatry. 1986;143(3):329-34.

11. Lewis CA. Cultural stereotype of the effects of religion on mental health. Br J Med Psychol. 2001;74(Pt 3):359-67.

12. Paloutzian R, Ellison C. Loneliness, spiritual well-being and the quality of life. In: Peplau D, Perlman D. Loneliness: a sourcebook of current theory, research and therapy. New York: John Wiley and Sons; 1982. p. 22435.

13. Mari JJ, Williams P. A validity study of a psychiatric screening questionnaire (SRQ-20) in primary care in the city of São Paulo. Br J Psychiatry. 1986;148:23-6.

14. Grabovac $A D$, Ganesan S. Spirituality and religion in Canadian psychiatry residency training. Can J Psychiatry. 2003;48(3):171-5.

15. Paiva GJ. Representação social da religião em docentespesquisadores universitários. Psicol USP. 1999;10(2):227-39.

16. Freud S. Totem e tabu. Rio de Janeiro: Imago; 1974. v. 13.

17. Dantas CR, Pavarin LB, Dalgalarrondo P. Sintomas de conteúdo religioso em pacientes psiquiátricos. Rev Bras Psiquiatr. 1999;21(3):158-64.

18. Fallot RD. The place of spirituality and religion in mental health services. New Dir Ment Health Serv. 2001;Fall(91):79-88.

\section{RESUMO}

Introdução: Religiosidade/espiritualidade e saúde mental parecem positivamente associadas. $O$ estudo examina associações entre bem-estar espiritual e distúrbios psiquiátricos menores em estudantes de Psicologia.

Métodos: Uma escala de bem-estar espiritual, o Self-Reporting Questionnaire (SRQ-20) e informações sociodemográficas foram utilizadas na totalidade $(n=$ 351) dos alunos de Psicologia da Universidade Católica de Pelotas (RS), no ano de 2002. Foram comparados os escores atuais com os dos alunos de Direito e Medicina da mesma universidade em 2001 $(n=464)$.

Resultados: $84,6 \%$ dos alunos de Psicologia apresentaram espiritualidade negativa, comparados a $68,8 \%$ dos acadêmicos de Medicina e $68,5 \%$ de Direito $(p<0,001)$. Diferenças semelhantes foram encontradas nas subescalas de bem-estar existencial e religioso. O SRQ-20 foi similar nos três cursos. A razão de chances mostrou associação de 3,71 (IC 95\% 1,29-10,68) entre o bem-estar espiritual e o escore do SRQ-20 em alunos de Psicologia. 
Discussão: Os achados coincidem com a experiência internacional. É preocupante que estudantes de Psicologia se mostrem mais distantes de questões espirituais/religiosas, levando em conta a associação espiritualidade/saúde-enfermidade.

Conclusão: A religiosidade/espiritualidade se mostrou como fator inversamente associado a transtornos psiquiátricos menores em estudantes de Psicologia.

Descritores: Espiritualidade, religiosidade, alunos de Psicologia, saúde mental, transtornos psiquiátricos menores.

\section{ABSTRACT}

Introduction: Religiosity/spirituality and mental health seem to be positively associated. This study examines associations between spiritual well-being and psychiatric disorders in psychology students.

Methods: A spiritual well-being scale - the selfreporting questionnaire (SRQ-20) - and sociodemographic information were used to all psychology students $(n=351)$ from Universidade Católica de Pelotas, RS, Brazil, during the year of 2002. Current scores were compared to those obtained from medical and law students of the same university in 2001 ( $n=464)$.

Results: $84.6 \%$ of psychology students presented negative spirituality, compared to $68.8 \%$ of medical and $68.5 \%$ of law students $(p<0.001)$. Similar differences were found in the existential and religious subscales. The SRQ-20 was similar in all three courses. The odds ratio showed an association of 3.71 (95\% Cl 1.29-10.68) between spiritual wellbeing and the SRQ-20 scores of psychology students.

Discussion: The findings coincide with the international experience. It is a matter of concern that psychology students define themselves more distant from spiritual/religious issues, considering the association spirituality/health-illness.

Conclusion: Spirituality-religiosity appeared as a factor inversely associated to minor psychiatric disorders among psychology students.

Keywords: Spirituality, religiosity, psychology students, mental health, minor psychiatric disorders. Title: Spiritual well-being and minor psychiatric disorders in psychology students: a cross-sectional study

\section{RESUMEN}

Introducción: Religiosidad/espiritualidad y salud mental parecen positivamente asociadas. El estudio examina asociaciones entre bienestar espiritual y trastornos psiquiátricos menores en estudiantes de Psicología.

Métodos: Una escala de bienestar espiritual (SWBS), el SRQ-20 y datos demográficos han sido utilizados en la totalidad $(n=351)$ de alumnos de Psicología de la Universidad Católica de Pelotas, RS, Brasil, en el año 2002. Se han comparado los rangos actuales con aquellos de los alumnos de Derecho y Medicina de la misma universidad, en $2001(n=464)$.

Resultados: $84,6 \%$ de los estudiantes de Psicología presentaron espiritualidad negativa, comparados con $68,8 \%$ en Medicina y $68,5 \%$ en Derecho $(p<0,001)$. Análogas diferencias se han encontrado en las subescalas de bienestar existencial y religioso. EI SRQ fue similar en los tres cursos. La razón de chances ha mostrado asociación de 3,71 (IC 95\% 1,29-10,68) entre bienestar espiritual y el rango del SRQ en los alumnos de Psicología.

Discusión: Los hallazgos coinciden con la experiencia internacional. Es preocupante que estudiantes de Psicología se muestren más distantes de cuestiones espirituales/religiosas, teniendo en cuenta la asociación espiritualidad - salud/ enfermedad.

Conclusión: La religiosidad-espiritualidad se mostró como factor inversamente asociado a trastornos psiquiátricos menores en estudiantes de Psicología.

Palabras clave: Espiritualidad, religiosidad, estudiantes de Psicología, salud mental, trastornos psiquiátricos menores.

Título: Bienestar espiritual y trastornos psiquiátricos menores en estudiantes de Psicología: estudio transversal

Correspondência:

Marina Gastaud

Mestrado em Saúde e Comportamento

Rua Almirante Barroso 1202, Bloco G, 107

CEP 96010-280 - Pelotas - RS

E-mail: marinagastaud@hotmail.com

Copyright (c) Revista de Psiquiatria

do Rio Grande do Sul - SPRS 